\title{
Antenna Optimization Using Multiobjective Algorithms
}

\author{
X. L. Travassos, ${ }^{1}$ D. A. G. Vieira, ${ }^{2}$ and A. C. Lisboa ${ }^{2}$ \\ ${ }^{1}$ Electromagnetic Devices Laboratory, SENAI-Integrated Centre of Manufacture and Technology, 41650-050 Salvador, BA, Brazil \\ ${ }^{2}$ ENACOM-Handcrafted Technologies, 31310-260 Belo Horizonte, MG, Brazil
}

Correspondence should be addressed to D. A. G. Vieira, douglas.vieira@enacom.com.br

Received 29 October 2011; Accepted 5 December 2011

Academic Editors: I.-S. Koh and B.-H. Soong

Copyright ( 2012 X. L. Travassos et al. This is an open access article distributed under the Creative Commons Attribution License, which permits unrestricted use, distribution, and reproduction in any medium, provided the original work is properly cited.

\begin{abstract}
This paper presents several applications of multiobjective optimization to antenna design, emphasizing the main general steps in this process. Specifications of antennas usually involve many conflicting objectives related to directivity, impedance matching, cross-polarization, and frequency range. These requirements induce multiobjective problems, which are formulated, solved, and analyzed here for three distinct antenna designs: a bowtie antenna for ground-penetrating radars, a reflector antenna for satellite broadcast systems, and a meander-line antenna for radio-frequency identification tags. Both stochastic and deterministic methods are considered in the analysis.
\end{abstract}

\section{Introduction}

Antenna optimization aims at creating advanced and complex electromagnetic devices that must be competitive in terms of performance, serviceability, and cost effectiveness. This process involves selection of appropriate objective functions (usually conflicting), design variables, parameters, and constraints. In most antenna optimization problems, several goals must be satisfied simultaneously in order to obtain an optimal solution. As these objectives are often conflicting, no single solution may exist that is best regarding all considered goals. In many situations, antenna optimization can be viewed as a multidisciplinary engineered problem. However, most of the problems can be divided into search for optimal solutions and approximate solution of Maxwell's equations using numerical methods.

Important features regarding antenna optimization have to be considered: the objective function might have several local minima and its evaluation can be expensive. Of course, trying to find the global minimum can be prohibitive; therefore, using a suitable method for the given problem is fundamental for a proper engineering solution.
For instance, some problems have well-established engineering solutions that were achieved after several years of tests and experiments. In such a situation, it may be desired to find a novel design which improves the known standard result, even though it may not be a global minimum. When the gradient or at least one subgradient is known, there are a couple of deterministic methods which can guarantee improvement (if an improved solution exists) of a given result [1].

Nevertheless, conditions where deterministic methods work well may not be achieved and, thus, stochastic methods may become a good alternative. In fact, stochastic methods such as genetic algorithms, particle swarm optimization, and immune systems are known to be very robust and capable of finding good results. However, they are also known to have slow convergence; that is, they require a high number of oracle queries. Solving antenna optimization problems is, therefore, a conflicting problem where fast methods only carry local guarantees while robust methods are prone to have very slow convergence. The aim of this paper is to present some antenna optimization problems and clarify some of the main issues related to the choice of optimization algorithms for antenna design. 
Multiobjective optimization and its application to antennas for ground penetrating radar (GPR), satellite broadcast communications, and RFID tags are the subject of this work.

\section{Antenna Designs with Multiple-Objective Optimization}

A trial and error process is often used for antenna design, where the designer must have considerable experience and intuition. This type of design has been subject to fundamental changes. Modern antenna systems are no longer designed on a simple desk using tables and calculators with inevitable design errors. Today, the design is computer aided, and both highly complex antennas and complete electronic systems are simulated together. In this context, optimization comes naturally as a designer's tool to produce an efficient and automatic "trial and error" process.

In order to formulate an optimization problem, degrees of freedom in the design must be properly identified and parameterized, and objectives and constraints for the design must be quantified given a set of parameters. A canonical query form for optimization problems is the extremum of quantities (e.g., maximum directivity, minimum standingwave ratio) given some bounds in quantities (e.g., upper bound on size, lower bound on amplitude gap between polarizations).

For problems with more than one objective, the concept of optimum becomes more complex, and it is qualified as Pareto optimum [2]. In this concept, for instance, the set of parameters where the directivity is maximum is not necessarily where the standing-wave ratio is minimum, so that tradeoffs between them become also an optimum in the sense that no point in the Pareto set has both objectives better than another point in it.

An antenna design usually involves specifications related to directivity, impedance matching, cross-polarization, and frequency range, which can be formulated as both objective or constraint functions. The types of these functions define the class of the optimization problem. In antenna design, evaluations of the computational model used in the optimization problem (i.e., evaluations of a black box problem, also called oracle queries) are, most of the time, expensive since they are based on numerical methods to approximate solutions of Maxwell's equations. Hence, the optimization speed is closely related to the number of oracle queries. Furthermore, there are theoretical guarantees of reaching global optima only for a few classes of problems, like linear and convex ones.

It is important to point out that the designer experience and intuition are still fundamental, and that optimization is only a tool. Good designers can define meaningful optimization problems, reduce the search space, and provide good starting points. Reducing search space not only speeds up the optimization but also potentially leads to problems with global optimality guarantees of solution. The design instances given hereinafter highlight and illustrate each one of these aspects.

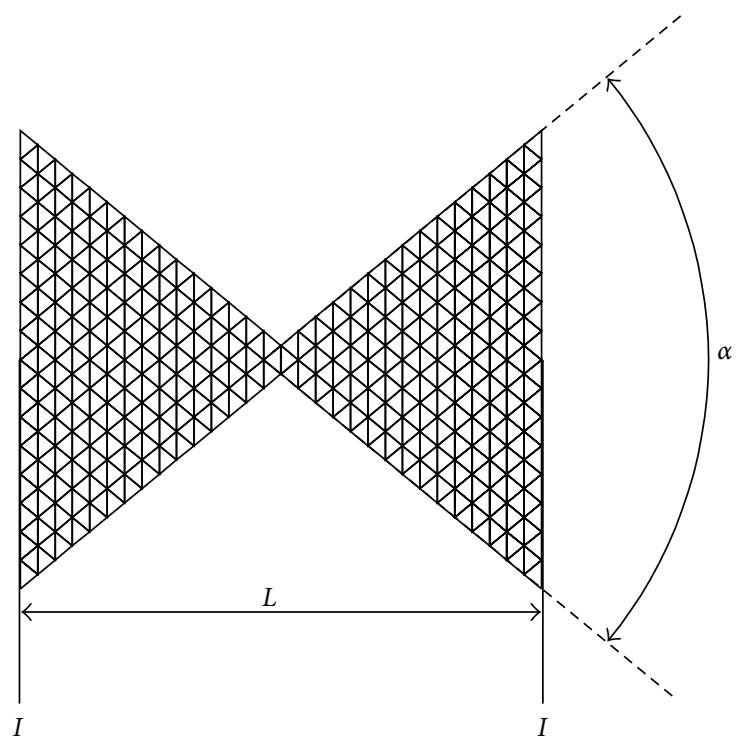

Figure 1: Geometry of the bowtie antenna.

\section{Design Instances}

3.1. Bowtie Antenna Design for Ground-Penetrating Radar. Most antennas for commercial ground-penetrating radar (GPR) are bowtie dipoles because of their light weight, low cost, and broadband characteristics. However, bowtie antennas have dipole-like omnidirectional patterns with broad main beams perpendicular to the plane of the antenna. Consequently, the image created by a GPR assessment could not correspond to the actual target, and closely spaced objects cannot be detected separately.

A multiobjective optimization problem to design more efficient bowtie dipole antennas can be formulated as $[3,4]$

$$
\begin{aligned}
& \text { minimize } f(x)=\left[\begin{array}{c}
-G_{\max }(x, v) \\
A(x)
\end{array}\right] \\
& \text { subject to } g(x)=A_{\min }-A(x) \leq 0, \\
& x_{\min } \leq x \leq x_{\max },
\end{aligned}
$$

where $x \in \mathbb{R}^{n}$ is the vector of design variables, $v$ is the frequency, $G_{\max }$ is the antenna gain, and $A(x)$ is the metal area. The objective of this problem is to minimize the metal area and to maximize the gain in the plane perpendicular to the antenna, with also a lower bound on metal area.

The bowtie antenna geometry (see Figure 1 and Table 1) was parameterized by length $L$, flare angle $\alpha$, and presence/absence of triangular elements $b \in\{0,1\}^{c^{2}-1}$, where $c \in$ $\{2,3, \ldots\}$ is the triangular grid density. All these parameters are design variables. The binary variable $b$ behaves like a topological geometric degree of freedom for the problem. It also introduces integer constraints to the problem. These constraints cannot be relaxed (i.e., $b \in\{0,1\}^{c^{2}-1}$ cannot be simulated), which makes stochastic algorithms more suitable than deterministic ones to solve the problem.

The optimal antenna suggested by a multiobjective genetic algorithm (MGA) for $v=1 \mathrm{GHz}, A_{\min }=0.8 A_{\max }$ 
TABLE 1: Optimization problem variables.

\begin{tabular}{lccc}
\hline & \multicolumn{3}{c}{ Parameter } \\
& $L(\lambda)$ & $\alpha\left({ }^{\circ}\right)$ & $R(\%)$ \\
\hline Min & 0.10 & 30 & 0 \\
Max & 1.00 & 120 & 20 \\
Opt & 0.87 & 79 & 11 \\
\hline
\end{tabular}

(i.e., $R=20 \%$ ), and $c=16$ was $\alpha=79^{\circ}, L=26 \mathrm{~cm}$, and $R=$ $11 \%\left(A_{\min }, A_{\max }, c, L, R\right)$ of elements removed (see Table 1$)$. The gain obtained in the plane normal to the antenna was $6.37 \mathrm{~dB}$ against $3.40 \mathrm{~dB}$ of the respective standard bowtie antenna (i.e., $b=1$ ), with an improvement of the halfpower beam width from $57.6^{\circ}$ to $43.2^{\circ}$. This result is the one whose metal area is maximal among a Pareto set with about 80 elements. Other solutions can be used according to the designer's needs. This approximate Pareto optimal set took about 2,500 oracle queries within 50 iterations of the MGA. A notable feature of stochastic algorithms is their ability to naturally provide a Pareto set as a result, instead of a single point.

During optimization, the method of moments (MoMs) was used to analyze the bowtie antenna. To validate the optimization result, a near field calculation was performed with the finite element method (FEM). FEM is slower and less accurate than MoM for far field, but it can handle complex geometries and material inhomogeneities accurately in the near field [3]. Therefore, FEM was chosen to perform an investigation considering the coupling effects of a geometrically complex antenna over an inhomogeneous dielectric. Moreover, a conductor shield was added to the antenna to improve directivity. The goal was to verify the behavior of a nondestructive assessment to detect the presence of a conducting bar buried $15 \mathrm{~cm}$ in the concrete and located parallel to the direction of the antenna. The changes in the antenna's input impedance were studied for three different scenarios.

3.2. Reflector Antenna Design for Satellite Coverage. Satellite coverage problems query for an antenna whose radiation pattern is as close as possible to a given specification. Many requirements can be specified other than maximum directivity inside the target area, like broadband operation, low cross-polarization, or maximum energy confinement inside the target area. They can be formulated as objective functions or constraint functions.

To formulate optimization problems, sample points are uniformly spread all over the target, so that good measures of coverage (mean gain inside target, $G_{\mathrm{av}}$ ) and energy confinement (energy ratio inside target $E_{\text {in }}$ ) can be taken. These measures are distinct for each sample frequency. Ideally, coverage also means energy confinement since illuminating a target implicitly means only that target is covered. However, due to physical constraints, transition to outside regions is not discontinuous and energy confinement has to be formulated separately. A multiobjective optimization

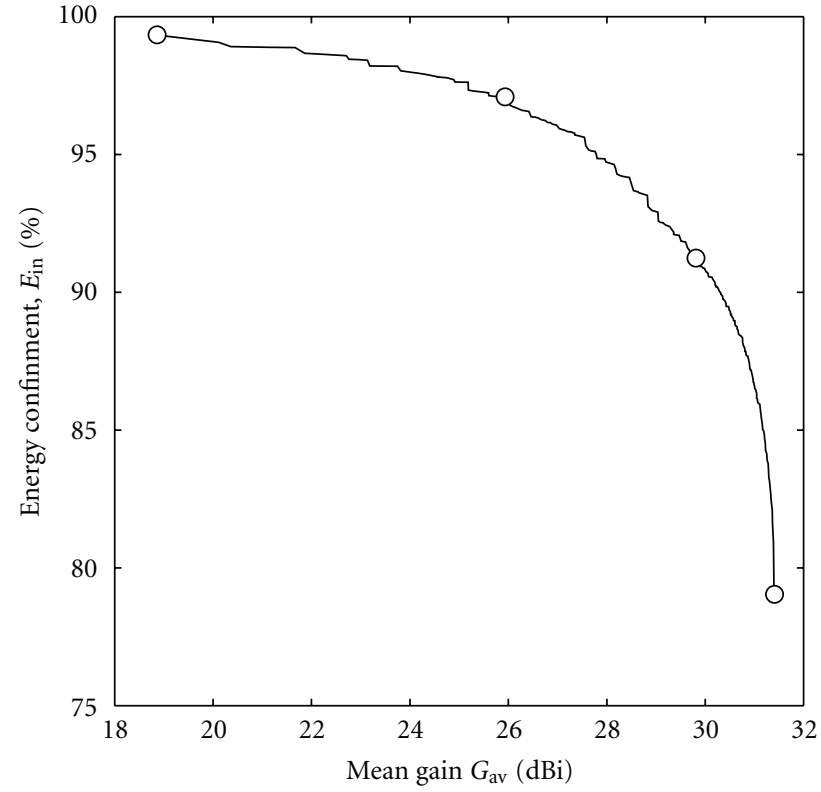

Figure 2: Pareto front for coverage and energy confinement. Radiation patterns for marked points are given in Figure 3.

problem formulation for satellite coverage with requirements on frequency range, coverage and energy confinement can be written as $[1,5,6]$

$$
\begin{aligned}
& \text { minimize } f(x)=\left[\begin{array}{l}
-G_{\mathrm{av}}(x, v) \\
-E_{\mathrm{in}}(x, v)
\end{array}\right] \\
& \text { subject to } x_{\min } \leq x \leq x_{\max },
\end{aligned}
$$

where $x \in \mathbb{R}^{n}$ is the vector of design variables, and $\nu \in \mathbb{R}^{o}$ is the vector of sample frequencies. The set of objective functions is considered for each sample frequency. For example, 3 sample frequencies for coverage and energy confinement lead to an optimization problem with 6 objective functions.

Figure 2 shows the Pareto front of the coverage problem (2) for $o=1$ and $n=38$ [6], where the radiation patterns of points marked are shown in Figure 3. Each Pareto optimal point took about 295 oracle queries within 5 iterations of the cone of efficient directions algorithm (CEDA). The results consider a single-reflector single-feed offset reflector antenna with circular aperture, parameterized by 35 degrees of freedom on four cubic triangular Bézier patches [1] representing the reflector shape, and 3 parameters relative to feed rotation and position on the $x=0$ plane, as shown in Figure 4. This parameterization considers, as reference, a parabolic antenna with circular aperture of radius $R=$ $0.762 \mathrm{~m}$, focal distance $F=1.506 \mathrm{~m}$, and offset $H=1.245 \mathrm{~m}$ [5] (see respective radiation pattern in Figure 5).

Due to distance from target and power supply constraints, reflector antennas are the best choice since they can be highly directive and efficient. For a single reflector antenna, the expected shape is nearly parabolic, convex, and smooth. The parameterization used in the aforementioned results [1] tries to encompass all these features, but it is 


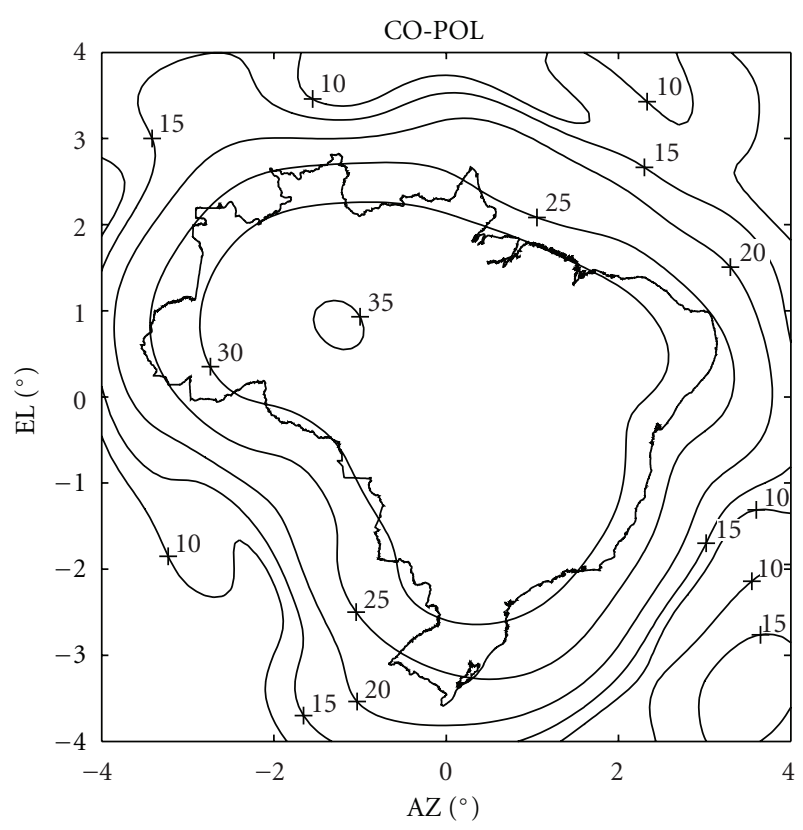

(a)

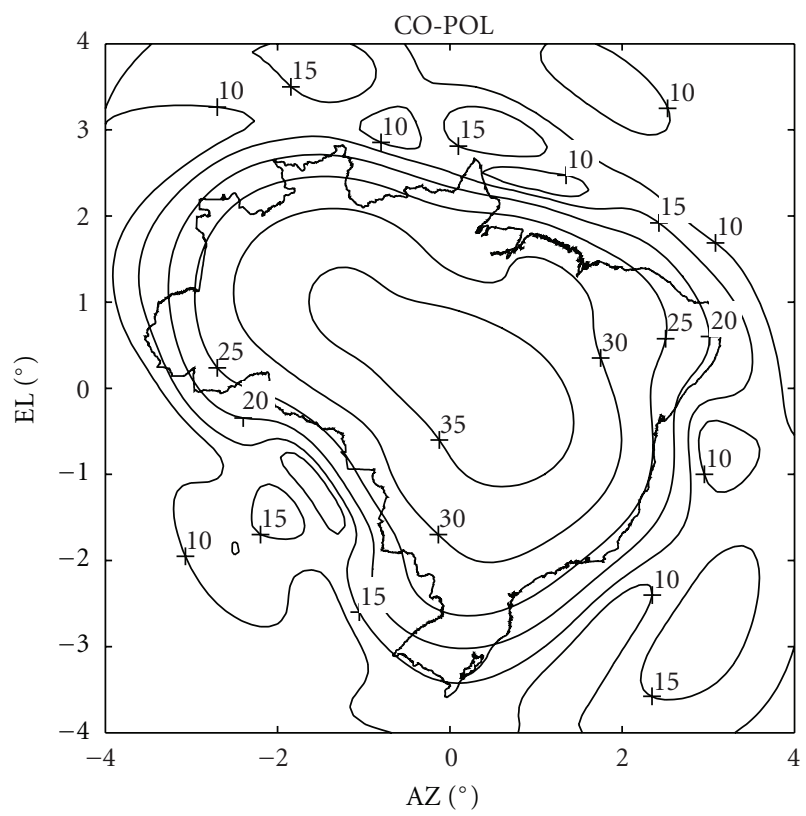

(c)

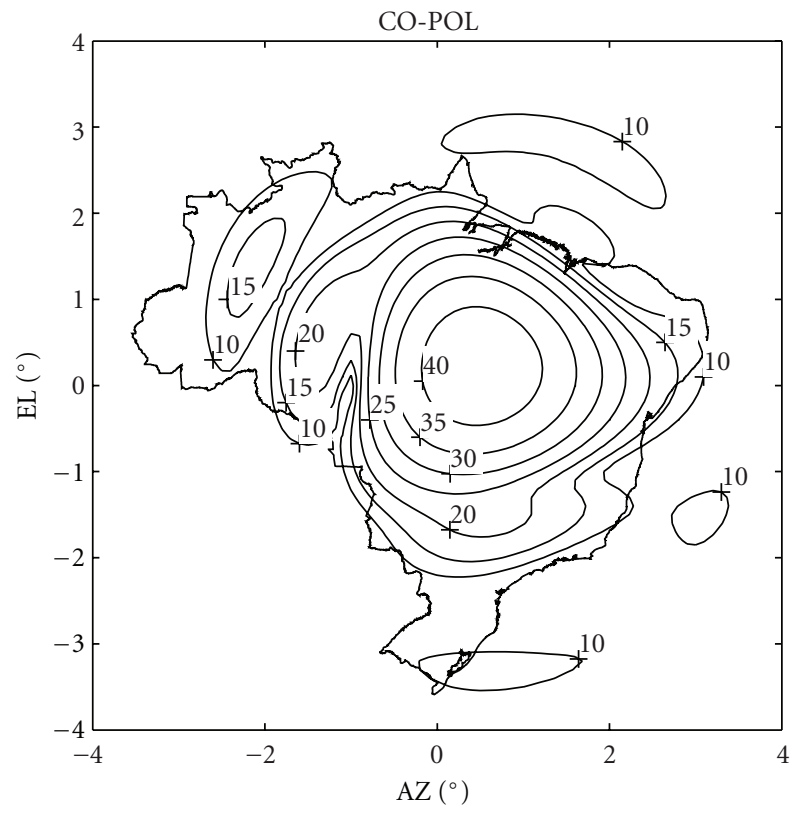

(b)

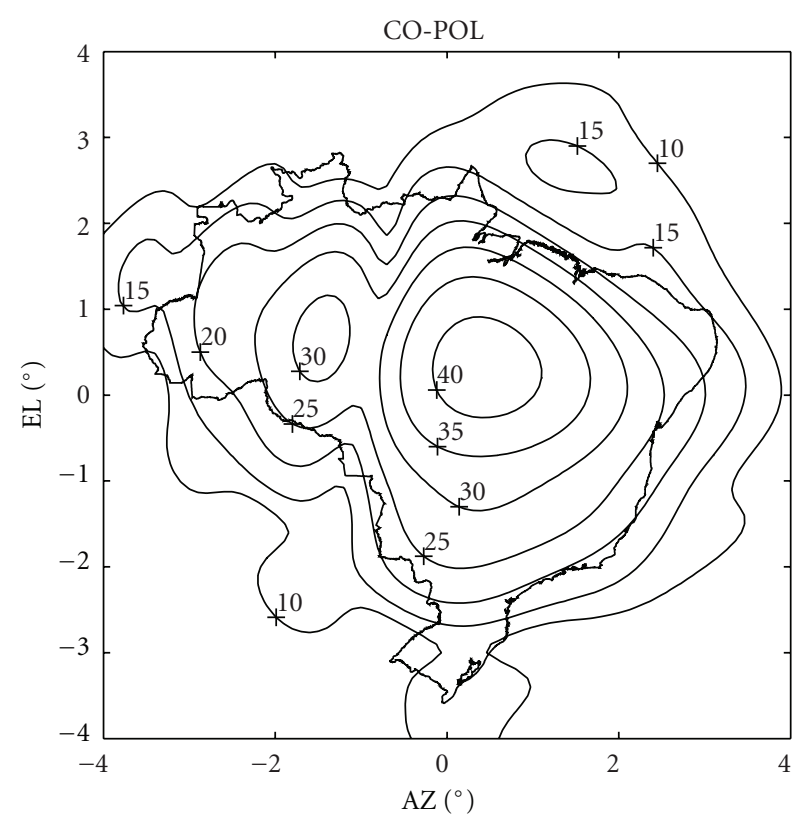

(d)

FIGURE 3: Radiation patterns for maximum coverage (a), maximum energy confinement (b), and intermediate tradeoffs between them (c, d).

quite extensive. A more elegant parameterization was used by Duan and Rahmat-Samii [5], but its truncated basis functions with trigonometric functions make the optimization problem harder to solve, especially because of higher multimodality in the objective functions, and the expected convex shape is more difficult to guarantee. A better choice would be orthogonal basis functions for convex functions.

Since the expected optimal antenna is smooth and greater than a few wavelengths, it can be quickly and accurately analyzed using physical optics (PO) approximation [7] applied to far field evaluations. The far-field integral for the current source from PO is solved using Gauss-Legendre numerical integration method.

One last point to consider is that CEDA presents a monotonic convergence, which means that all objectives are improved after every iteration. This notable ability implies a fast and robust convergence in practice and can be used to seed starting points for stochastic algorithms. Stochastic algorithms are in turn well suited to map Pareto sets and skip bad local minima, which makes them suitable for seeding starting points for deterministic algorithms. This cyclic relationship suggests a hybrid approach, which has 

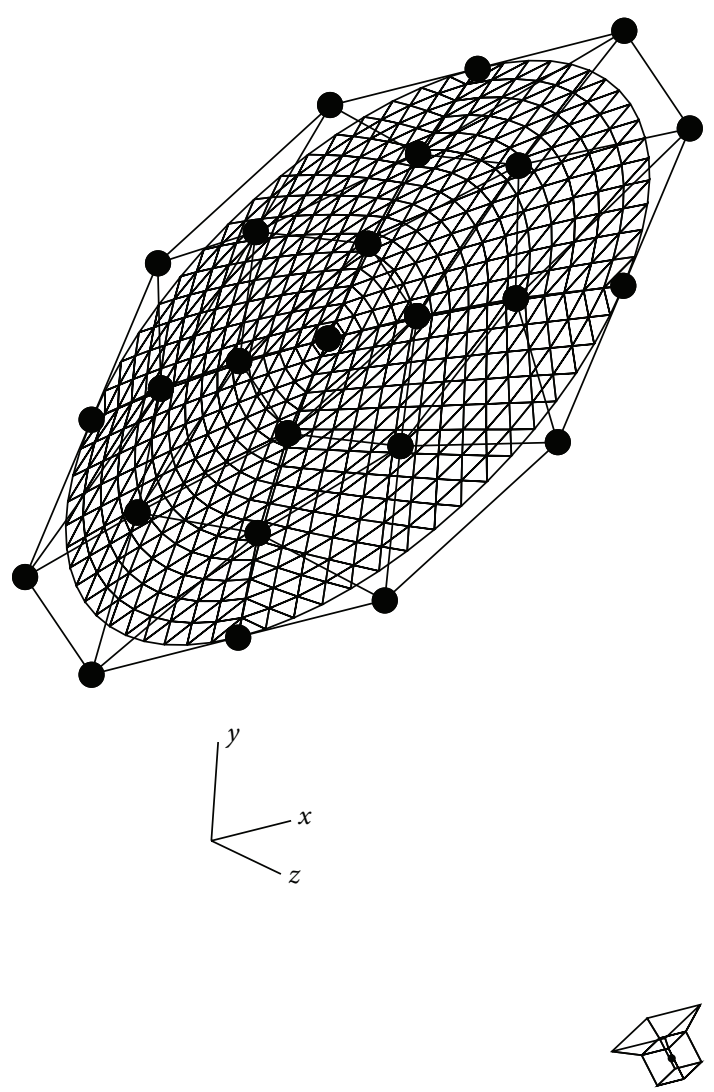

FIgure 4: The reflector antenna is parameterized by reflector shape and feed position and rotation on plane $x=0$. The reflector shape is defined by a Bézier surface whose parameters are the 25 control points (shown in dark dots) of a triangular control mesh.

already been used in literature, especially under the recent concept of memetic algorithms.

\subsection{Optimal Design of Meander-Line Antennas for Radio-} Frequency Identification. The low cost of electronic microcircuits and their low power consumption have made the development of identification systems through radio frequency practical, especially since the decade of the 1990s. Radio frequency identification (RFID) has been applied for tracking of products, luggage, books, animals, and other items where the tags can be attached to the objects, injected under the skin or embedded within items [8].

The RFID total market value in 2009 grew to $\$ 5.56$ billion, of which $\$ 2.18$ billion was spent only on passive tags alone [9]. Therefore, optimizing radio frequency identification systems has become crucial in improving the productivity and lowering costs in industry and supply chains. An RFID system consists basically in a receiver (tag), an emitter (reader), and a computational system (direct link). The tags have two main structures: the microchip, which provides the necessary power to transmit and receive information, and the antenna. The tags can be active, passive, or semiactive, depending on the mechanism of powering the microchip and transmitting information. Active tags have a local power source and electronics for performing

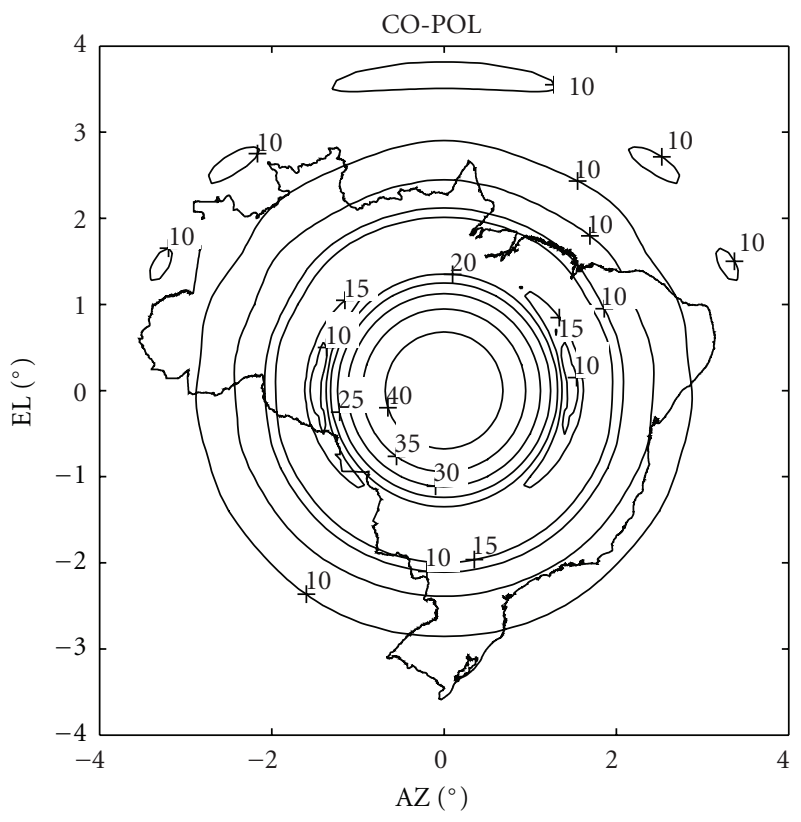

FIGURE 5: Radiation patterns for the reference parabolic reflector antenna.

specialized tasks [10]. The type of RFID tags chosen is based on the specific application. For low-cost tags and relatively short read ranges (around $3 \mathrm{~m}$ [11]), passive tags are most appropriate.

Due to the diversity of materials and packages that need to be identified, tag antennas development for passive UHF RFID systems has become challenging. The performance of the tag antennas is very dependent on the properties of the objects which the tags are attached to. The material of the objects can influence the capacitive characteristics of the tag antenna as well as modify its radiation pattern. Many studies have been carried out in order to investigate these effects in tags attached to metallic surfaces and water $[12,13]$ and to dielectrics $[14,15]$. An effort to find the best optimized tag antenna for a given application improves the performance of the entire RFID system, because it can increase the power transfer coefficient and the read range.

The design in this paper was motivated by an application in the coffee industry. In this business, the product is stored in sacks in the producers' farms and needs to be transported to the local cooperatives. Each cooperative receives coffee sacks from different producers, normally using treadmills, and the receiving process demands time and labor force and is usually inefficient. In addition, the origin of the coffee must be traced until it reaches the final consumer and the material flow must be controlled in real time. In this context, RFID systems can be used to improve the productivity and competitiveness and reduce costs.

Meander-line antennas are one of the most commonly used in UHF RFID tags, mainly because of their tunability and size. Several papers have been published on RFID meander-line antenna design. Some articles have sought improved antenna gains and small size by using different configurations of meander-line antennas for passive RFID, which were explored applying genetic algorithm 
TABLE 2: Optimization problem variables for multiobjective formulation.

\begin{tabular}{ccccccccccc}
\hline & \multicolumn{10}{c}{ Parameter $(\mathrm{mm})$} \\
& $w$ & $b$ & $a_{1}$ & $a_{2}$ & $a_{3}$ & $a_{4}$ & $s_{1}$ & $s_{2}$ & $s_{3}$ & $s_{4}$ \\
\hline Max & 0.50 & 12.60 & 1.00 & 1.00 & 1.00 & 1.00 & 1.00 & 1.00 & 1.00 & 1.00 \\
Min & 0.80 & 21.00 & 7.00 & 7.00 & 7.00 & 7.00 & 7.00 & 7.00 & 7.00 & 7.00 \\
Opt & 0.78 & 15.11 & 4.70 & 3.98 & 6.42 & 5.66 & 3.80 & 1.42 & 6.00 & 3.77 \\
\hline
\end{tabular}

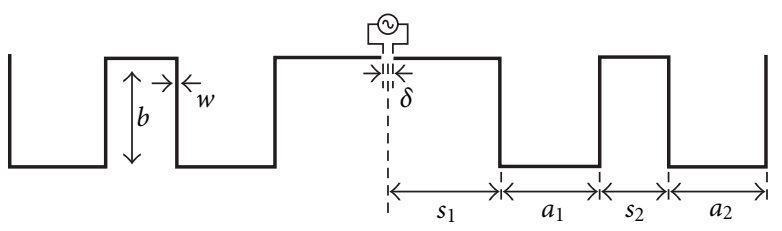

Figure 6: Geometry of the meander antenna for $c=2$.

(GA) optimization and the method of moments (MoMs) $[16,17]$. In [15], a loaded meander-line tag antenna was designed focusing on a specific application: box tracking in warehouses; the box content and the sensitivity to fabrication process were also considered.

Some methods have been used to characterize impedances and design meander-line antennas [18], including multiobjective optimization [19], and GA has been shown to be an efficient optimization tool for selecting globally optimal parameters of the antenna [20]. This paper introduces design techniques using optimization in order to cope with operation inside a frequency range.

3.3.1. Model and Parametrization. The meander-line antenna geometry (see Figure 6) is parameterized by the number of meanders $c$ on each side, feed gap, trace width $w$, height $b$, meander step length $a_{i}$, and spacing between meanders $s_{i}$, $i=1, \ldots, c$.

The antenna is discretized into regular quasiregular triangles and analyzed using a method of moments with a voltage gap feed assuming that $\delta=0$. The implementation was validated with classical antenna simulation software.

The parameters other than $\delta$ that compose the design variables are

$$
x=\left[\begin{array}{c}
w \\
b \\
a_{1} \\
\vdots \\
a_{c} \\
s_{1} \\
\vdots \\
s_{c}
\end{array}\right]
$$

which are variables (degrees of freedom) to be optimized in order to attend some given objectives.

3.3.2. Optimization Problem. A meander-line antenna cannot provide high directivity. This is a desired behavior considering that isotropic radiation would not be ideal for
TABLE 3: Specifications and optimal antenna profile.

\begin{tabular}{lc}
\hline Parameter & Value \\
\hline$L_{\min }(\mathrm{mm})$ & 10 \\
$L_{\max }(\mathrm{mm})$ & 100 \\
$\nu_{\min }(\mathrm{MHz})$ & 902 \\
$\nu_{\max }(\mathrm{MHz})$ & 928 \\
$Z_{\mathrm{s}}(\Omega)$ & $27.4-200.9 i$ \\
\hline
\end{tabular}

the application. The remaining features of interest are input impedance and size, which are used in the multiobjective optimization problem formulation:

$$
\begin{aligned}
& \text { minimize } f(x)=\operatorname{SWR}(x, v) \\
& \text { subject to } g(x)=\left[\begin{array}{l}
L_{\min }-L(x) \\
L(x)-L_{\max }
\end{array}\right] \leq 0, \\
& x_{\min } \leq x \leq x_{\max },
\end{aligned}
$$

where $x \in \mathbb{R}^{n}$ is the vector of design variables (4), $n=2+$ $2 c$, SWR is the standing wave ratio, $L$ is the antenna length, and $\nu \in \mathbb{R}^{o}$ is the vector of sample frequencies. There is one objective function for each sample frequency, hence a total of o objective functions. The optimization problem (5) queries for antennas with minimum SWR in each sample frequency and whose overall length and design variables lie inside an interval.

To optimize the worst case SWR inside the frequency range, the mono-objective optimization problem formulation

$$
\begin{aligned}
& \text { minimize } f(x, t)=t \\
& \text { subject to } g(x, t)=\left[\begin{array}{c}
\operatorname{SWR}(x, v)-t \\
L_{\min }-L(x) \\
L(x)-L_{\max }
\end{array}\right] \leq 0 \\
& x_{\min } \leq x \leq x_{\max }
\end{aligned}
$$

can be derived, where $t$ is the worst case SWR.

3.3.3. Optimization Results. The optimal variable results of (5) for $c=4$ and $o=5$ sample frequencies inside the frequency range are shown in Table 2 . The target impedance, frequency range and length constraints are given in Table 3. The length was $71.51 \mathrm{~mm}$ and the worst standing wave ratio (SWR) was 4.04 for the optimal antenna. SWR behavior inside the frequency range is shown in Figure 7. The result took 395 problem oracle queries within 9 iterations of the multiobjective deterministic algorithm with monotonic convergence [21].

In order to compare the two formulations (multiobjective and worst case), the CEDA algorithm was limited to 395 oracle queries to optimize (8) for $c=4, o=5$, and settings in Table 3, whose optimal results are shown in Table 4 . The length was $62.67 \mathrm{~mm}$ and the worst standing wave ratio (SWR) was 3.99 for the optimal antenna. SWR behavior inside the frequency range is shown in Figure 8. 


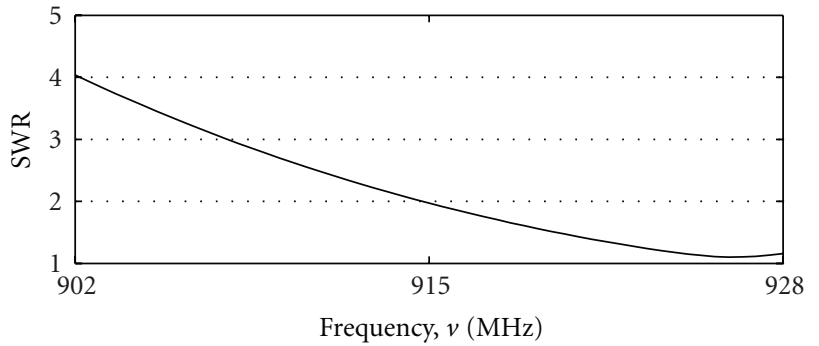

FIGURE 7: Standing wave ratio behavior inside frequency range.

TABLE 4: Optimization problem variables for worst case formulation.

\begin{tabular}{ccccccccccc}
\hline & \multicolumn{10}{c}{ Parameter $(\mathrm{mm})$} \\
& $w$ & $b$ & $a_{1}$ & $a_{2}$ & $a_{3}$ & $a_{4}$ & $s_{1}$ & $s_{2}$ & $s_{3}$ & $s_{4}$ \\
\hline Max & 0.50 & 12.60 & 1.00 & 1.00 & 1.00 & 1.00 & 1.00 & 1.00 & 1.00 & 1.00 \\
Min & 0.80 & 21.00 & 7.00 & 7.00 & 7.00 & 7.00 & 7.00 & 7.00 & 7.00 & 7.00 \\
Opt & 0.60 & 15.81 & 5.95 & 2.26 & 3.82 & 1.94 & 4.39 & 3.13 & 4.25 & 6.09 \\
\hline
\end{tabular}

The best SWR of about 1 was verified inside a frequency range of $1.75 \%$ for optimal 4-meander antennas, even though the worst SWR is about 4 . More degrees of freedom could be considered in order to improve worst cases (e.g., increasing the number of meanders or parametrizing each meander height individually).

The multiobjective formulation converges faster than the worst-case formulation. Furthermore, the theoretical guarantee of always improving all objective functions after each iteration may be very suitable in real world applications. However, considering that SWRs at sample frequencies of 1 and 40 could be considered Pareto optimal for the former formulation, results for the worst case formulation may be regarded more meaningful.

\section{Conclusion}

Multiobjective design of antennas is an important and active field of inquiry. Many important problems, such as those presented in this paper, have been considered in the last few years. The approaches discussed in this paper are conceptually different. Evolutionary optimization relies on global search for the best solution. Even though they are robust techniques, they depend on some user's defined parameters and the convergence may be slow. Deterministic methods have asymptotic fast convergence. However, they are prone to getting trapped in local minima. In practice, the choice of the optimization algorithm will depend on the nature of the problem and the designer's experience.

\section{Acknowledgments}

This work was supported by the National Counsel of Technological and Scientific Development (CNPq), Minas Gerais State Research Foundation (FAPEMIG), and Bahia State Research Foundation (FAPESB).

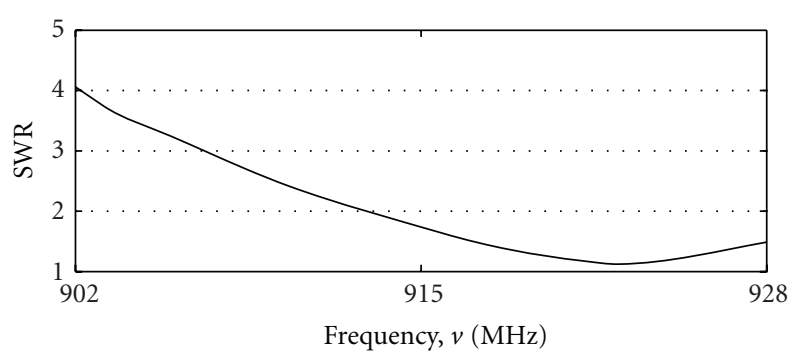

FIGURE 8: Optimal standing wave ratio behavior inside frequency range for worst case formulation.

\section{References}

[1] A. C. Lisboa, D. A. G. Vieira, J. A. Vasconcelos, R. R. Saldanha, and R. H. C. Takahashi, "Multiobjective shape optimization of broad-band reflector antennas using the cone of efficient directions algorithm," IEEE Transactions on Magnetics, vol. 42, no. 4, pp. 1223-1226, 2006.

[2] K. Deb, Multi-Objective Optimization Using Evolutionary Algorithms, John Wiley \& Sons, New York, NY, USA, 2002.

[3] X. L. Travassos, N. Ida, C. Vollaire, and A. Nicolas, "Solution of Maxwell's equations for the simulation and optimization of the radar assessment of concrete structures," Research in Nondestructive Evaluation, vol. 18, no. 3, pp. 151-161, 2007.

[4] X. L. Travassos, Modélisation numérique pour l'évaluation non destructive électromagnétique: application au contrôle non destructif des structures en béton, Ph.D. thesis, L'École Centrale de Lyon, Ecully, France, June 2007.

[5] D.-W. Duan and Y. Rahmat-Samii, "Generalized diffraction synthesis technique for high performance reflector antennas," IEEE Transactions on Antennas and Propagation, vol. 43, no. 1, pp. 27-40, 1995.

[6] A. C. Lisboa, D. A. G. Vieira, J. A. Vansconcelos, R. R. Saldanha, and R. H. C. Takahashi, "Decreasing interference in satellite broadband communication systems using modeled reflector antennas," IEEE Transactions on Magnetics, vol. 44, no. 6, pp. 958-961, 2008.

[7] C. A. Balanis, Advanced Engineering Electromagnetics, John Wiley \& Sons, 1989.

[8] S. Polniak, "The RFID case study book: RFID application stories from around the globe," Tech. Rep., Abhisam Software, 2007.

[9] P. Harrop, “Printed RFID in 2010," IDTechEx, 2010.

[10] Z. N. Chen, Antennas for Portable Devices, John Wiley \& Sons, 2007.

[11] D. M. Dobkin, The RF in RFID: Passive UHF RFID in Practice, Elsevier, 2008.

[12] N. A. Mohammed, M. Sivakumar, and D. D. Deavours, "An RFID tag capable of free-space and on-metal operation," type, Information and Telecommunications Technology Center, 2008.

[13] J. Y. Park and J. M. Woo, "Miniaturised dual-band S-shaped RFID tag antenna mountable on metallic surface," Electronics Letters, vol. 44, no. 23, pp. 1339-1341, 2008.

[14] J. D. Griffin and G. D. Durgin, "Radio link budgets for 915MHz RFID antennas placed on various objetcs," in Texas Wireless Symposium, vol. 44, Atlanta, Ga, USA, 2005.

[15] K. V. S. Rao, P. V. Nikitin, and S. F. Lam, "Antenna design for UHF RFID tags: a review and a practical application," IEEE Transactions on Antennas and Propagation, vol. 53, no. 12, pp. 3870-3876, 2005. 
[16] G. Marrocco, A. Fonte, and F. Bardati, "Evolutionary design of miniaturized meander-line antennas for RFID applications," in Proceedings of the IEEE Antennas and Propagation Society International Symposium, pp. 362-365, June 2002.

[17] G. Marrocco, "Gain-optimized self-resonant meander line antennas for RFID applications," IEEE Antennas and Wireless Propagation Letters, vol. 2, pp. 302-305, 2003.

[18] X. Qing, C. K. Goh, and Z. N. Chen, "Impedance characterization of rfid tag antennas and application in tag co-design," IEEE Transactions on Microwave Theory and Techniques, vol. 57, no. 5, pp. 1268-1274, 2009.

[19] X. L. Travassos, D. A. G. Vieira, A. C. Lisboa, M. M. B. Lima, and N. Ida, "Antenna optimization using multi-objective algorithms," in Proceedings of the 4th European Conference on Antennas and Propagation (EuCAP '10), April 2010.

[20] D. Zhou, R. A. Abd-Alhameed, and C. H. See, "Meanderline antenna design for UHF RFID tag using a genetic algorithm," in Proceedings of the Progress in Eletromagnetics Research Symposium, pp. 1253-1257, March 2009.

[21] D. A. G. Vieira, R. H. C. Takahashi, and R. R. Saldanha, "Multicriteria optimization with a multiobjective golden section line search," Mathematical Programming, vol. 131, no. 1-2, pp. 131-161, 2012. 

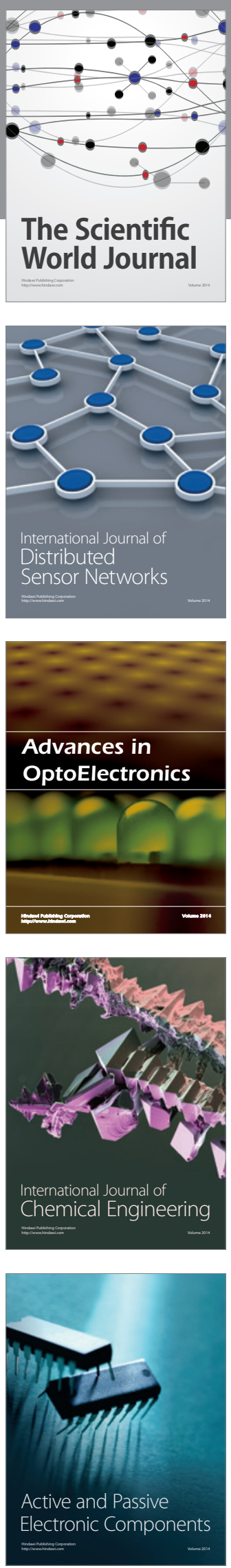
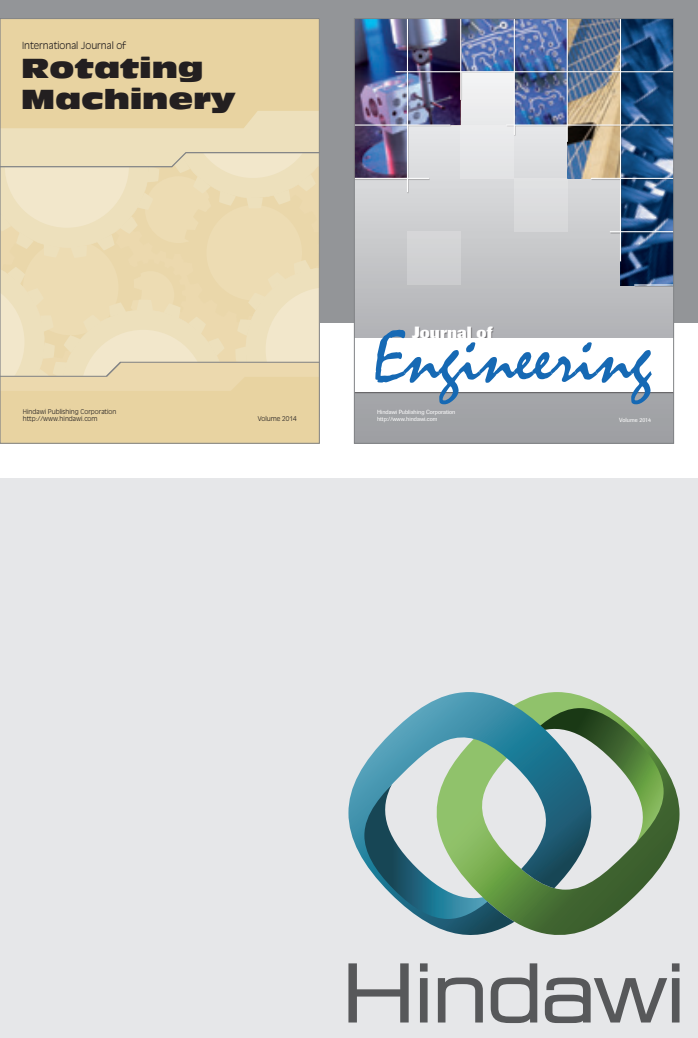

Submit your manuscripts at

http://www.hindawi.com
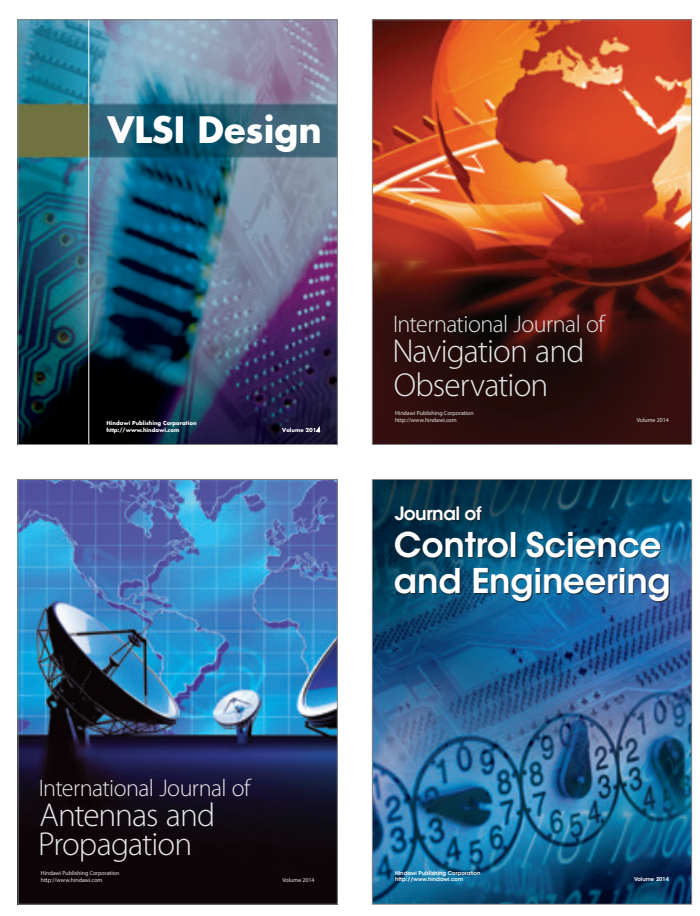
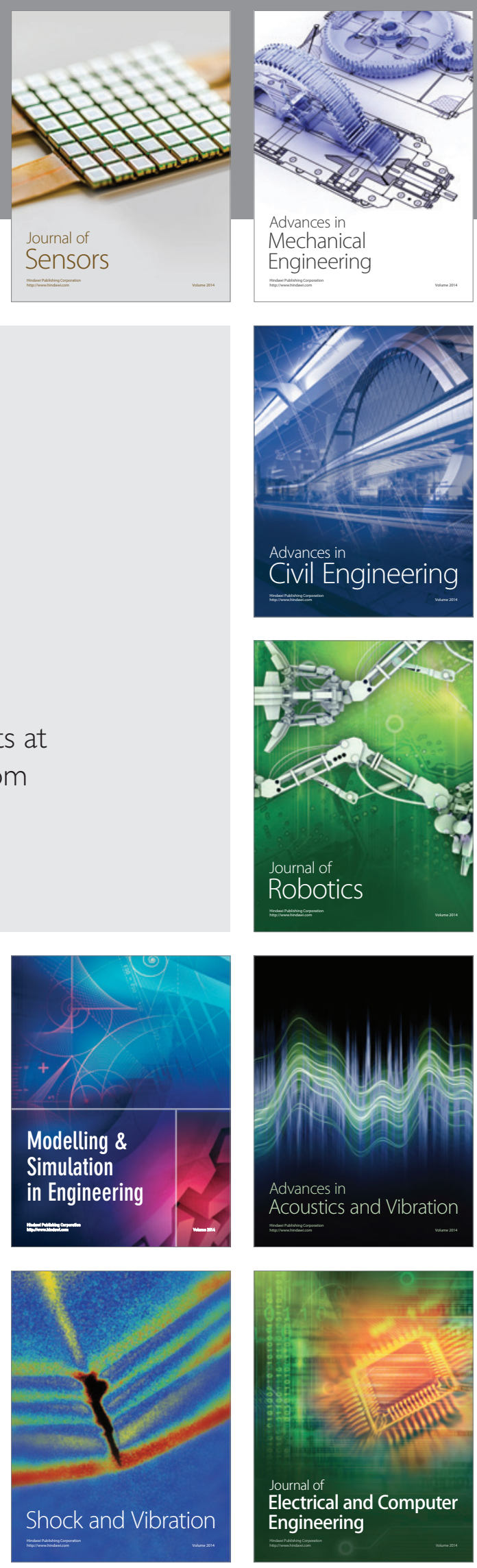